\title{
Transposition d'un rapport HTA de I'IQWiG' au contexte suisse
}

\author{
Les rapports des instituts étrangers de HTA peuvent-ils simplement être appliqués \\ au contexte suisse? Le conseil d'experts du Swiss Medical Board (SMB) a examiné \\ cette question sur la base d'un rapport de l'institut allemand pour la qualité et la \\ rentabilité du système de santé (Deutsches Institut für Qualität und Wirtschaftlich- \\ keit im Gesundheitswesen, IQWIG). Cette analyse représente un nouveau pas vers le \\ développement d'une méthode permettant d'exploiter les possibles synergies.
}

\author{
Conseil d'experts du \\ Swiss Medical Board*: \\ Eva Cignacco $^{a}$, \\ Peter Jüni ${ }^{b}$, Peter Meier-Abt ${ }^{c}$, \\ Urs Metzger, \\ Nikola Biller-Andorno ${ }^{e}$, \\ Stefan Felderf, Brigitte Tagg \\ a Institut des sciences \\ infirmières, Université \\ de Bâle (Médecine) \\ b Prof. d'épidémiologie \\ clinique, Université de Berne \\ (Médecine) \\ c Prof. em. de pharmacologie \\ clinique, vicerecteur \\ de l'Université de Bâle \\ (Médecine) \\ d Prof. ém. médecin chef en \\ chirurgie, Zurich (Médecine) \\ e Prof. d'éthique biomédicale, \\ Université de Zurich (Ethique) \\ f Prof. d'économie de la santé, \\ Université de Bâle \\ g Prof. de droit pénal, procé- \\ dure pénale et droit \\ de la médecine, \\ Université de Zurich (Droit)
}

\footnotetext{
* Organisation et composition sous www.medical-board.ch/ index.php?id=818\&L=1
}

\section{Correspondance:}

Susanna Marti Calmell

Secrétariat Swiss Medical Board

Obstgartenstrasse 21

CH-8090 Zurich

Tél. 0432592479

info[at]medical-board

www.swissmedicalboard.ch

\section{Résumé du rapport d'origine}

Le présent rapport a pour but de présenter le fonctionnement et la méthodologie de l'IQWiG $^{1}$ en comparaison avec le Swiss Medical Board (SMB) en vue de possibles synergies. Pour illustrer et concrétiser cette comparaison, on a examiné si un rapport récemment élaboré par l'IQWiG concernant la problématique d'un médicament précis (l'ézétimibe) dans le traitement de l'hypercholestérolémie peut être appliqué à un contexte suisse.

On entend par hypercholestérolémie un groupe de maladies qui s'accompagnent d'un taux de cholestérol sanguin continuellement et pathologiquement élevé. Cet état favorise, avec d'autres facteurs, les dépôts de lipoprotéines (plaques) sur les parois internes des artères (athéromatose). Les conséquences en sont de graves troubles vasculaires chroniques, mais aussi aigus (ischémies), tels qu'un infarctus du myocarde ou un accident vasculaire cérébral (AVC).

En résumé, on peut noter que les rapports de l'IQWiG mettent l'accent sur ce que l'on appelle $l^{\prime}$ «assessment» (analyse) et sur l'aspect de la valeur ajoutée et des inconvénients pour les patients. Le présent rapport s'est penché exclusivement, pour déterminer la valeur ajoutée et les inconvénients, sur des essais contrôlés randomisés (ECR). Les rapports de l'IQWiG ne se terminent pas par des recommandations d'actions, mais concluent en se demandant s'il existe, pour l'intervention examinée, une indication ou une preuve de valeur ajoutée (ou d'inconvénients) pour le patient.
En ce qui concerne l'utilisation des rapports de l'IQWiG comme base pour établir les rapports du Swiss Medical Board, les réflexions exposées conduisent aux recommandations suivantes:

- Les rapports de l'IQWiG peuvent être utilisés comme base pour l'élaboration du chapitre «Effets médicaux» des rapports du Swiss Medical Board si les conditions suivantes sont remplies.

- Concordance entre la question posée par le SMB et celle du rapport de l'IQWiG.

- Le rapport de l'IQWiG est d'actualité, c'est-àdire qu'il n'y a aucun élément indiquant que l'état des données a changé.

- Il existe suffisamment de données provenant des ECR avec des critères pertinents pour les patients pour que la prise en compte d'autres sources d'information (comme les études d'observation) ne soit pas nécessaire.

- Les chapitres sur les coûts, le rapport coût-efficacité et les réflexions éthiques et juridiques, ainsi que l'appréciation globale et des recommandations restent à élaborer.

1 L'Institut allemand pour la qualité et la rentabilité du système de santé publique (IQWiG) est un organisme scientifique indépendant qui examine les avantages et inconvénients des mesures médicales pour les patients. Il travaille à la demande du Comité fédéral conjoint (Gemeinsamer Bundesausschuss, G-BA), qui décide quelles prestations doivent être réalisées en Allemagne à la charge du régime légal d'assurance-maladie. 